\title{
Asymptotic behavior of the energetics and electronic structures of graphene with pyridinic defects
}

\author{
Mina Maruyama ${ }^{\mathrm{a}, *}$, Susumu Okada ${ }^{\mathrm{a}}$ \\ ${ }^{a}$ Graduate School of Pure and Applied Sciences, University of Tsukuba, 1-1-1 Tennodai, \\ Tsukuba, Ibaraki 305-8571, Japan
}

\begin{abstract}
Using density functional theory, we study the energetics and electronic structures of graphene with monovacancies, which are surrounded by three pyridinic $\mathrm{N}$ atoms, in terms of their density and arrangement. Our calculations demonstrated that the detailed geometric structure and the formation energy of the defects do not depend on the mutual arrangement of the defects but on their density. The electronic structure of graphene with defects, in contrast, is sensitive not only to the defect density but also to the defect arrangement whether or not the graphitic $\pi$ network that consists of $\mathrm{C}$ and $\mathrm{N}$ satisfies the Clar structure (full-benzenoide structure). Graphene with a defect that satisfies the Clar structure has a small energy gap between bonding and antibonding $\pi$ states at the $\Gamma$ point, while that without the Clar structure has a moderate gap between the bonding and antibonding $\pi$ states at the $\mathrm{K}$ point. Furthermore, the gap monotonically decreases with decreasing the defect density. A non-bonding $\pi$ state is induced at the Fermi level because of the sublattice imbalance of the graphitic $\pi$ network of $\mathrm{C}$ and $\mathrm{N}$ atoms, which implies that the $p_{z}$ state of pyridinic $\mathrm{N}$ atoms takes part in the $\pi$ electron
\end{abstract}

\footnotetext{
* Corresponding author

Email address: mmaruyama@comas.frsc.tsukuba.ac.jp (Mina Maruyama)
} 
network of $\mathrm{C}$ atoms. According to the extended nature of non-bonding states, its bandwidth slowly decreases upon increasing the defect-defect spacing.

\section{Introduction}

A honeycomb network of $\mathrm{sp}^{2}$ carbon atoms with single-atom thickness causes a pair of linear dispersion bands at the Fermi level [1, 2, 3], which results in a remarkable carrier mobility up to a few hundred thousand $\mathrm{cm}^{2} / \mathrm{Vs}$. This makes graphene an emerging material for the design of functional devices in the wide area of modern technologies [4]. In addition to the technological views, the linear dispersion bands lead to the unusual quantum Hall effect, which makes graphene a unique material for exploring the fundamental phenomena in a two-dimensional electron system [5]. Because of the bipartite network of $\pi$ electron distributed normal to the atomic layer, these remarkable electronic properties have been shown to be fragile towards external perturbations such as structural imperfections, structural corrugations, atom/molecule adsorptions, interactions with other graphene or substrates, and external electric field $[6,7,8,9,10,11]$. For example, graphene nanoribbons possess peculiar electronic structures depending on their width and edge atomic arrangements: graphene nanoribbons with zigzag edges possess edge localized states known as edge states caused by the delicate balance among the electron transfer around the edge atoms sites, while ribbons with armchair edges have either metallic or semiconducting properties depending on the discretized conditions imposed on graphene $[12,13,14,15,16,17,18,19]$. The topological defects, such as pentagons and heptagons, also cause substantial electronic structure modulation, even though all $\mathrm{C}$ atoms possess three-fold coordination, where all covalent bonds are fully saturated [20, 21, 22, 23]. In addition, atomic 
defects and pores occasionally cause a flat dispersion band at the Fermi level, owing to the sublattice imbalance of the bipartite networks of graphene, which leads to the spin polarization through the networks $[24,25]$. In addition to the defect structures, their mutual arrangements also affect the electronic structure around the Fermi level of graphitic materials.

Adsorptions or doping of foreign atoms are the other possible procedures to modify the electronic structure of graphene. An early theoretical work demonstrated that fluorinated graphene possesses a narrow dispersion band at the Fermi level, when F atoms are attached to one of two sublattices [26]. Even though the $\mathrm{F}$ atoms are only attached to particular sublattices, the fluorinated graphene no longer possesses the Dirac cone but massive valleys for both conduction and valence band edges when the graphitic region does not satisfy the resonant condition of aromaticity, as is the case of graphene with periodic vacancies [27, 28]. Substitutional doping of a borazine skeleton into graphene opens the finite band gap, which depends not only on the $\mathrm{BN}$ ring concentration but also on their mutual $\mathrm{BN}$ ring arrangements in the graphene network [29]. N-doped graphene also exhibits unusual electronic properties, which depend on the local and global atomic arrangements, and is applicable to electronic and catalytic devices [30, 31, 32, $33,34,35,36,37,38,39,40]$. In such devices, it is important to precisely tune the electronic structure near the Fermi level by $\mathrm{N}$ atom arrangements to tailor the device properties. The concentration and mutual arrangements of pyridinic $\mathrm{N}$ in graphene are essential for their catalytic applications, because the electron states associated with the non-bonding states of pyridinic $\mathrm{N}$ atoms are sensitive to their concentration and arrangements. However, the comprehensive and systematic studies of the energetics and electronic structure of graphene with pyridinic 
defects in terms of their arrangement and concentration are currently insufficient.

In accordance with the above background, in this work, we aim to investigate the energetics and electronic structure of graphene with monovacancy whose edges are terminated by $\mathrm{N}$ atoms with a pyridinic structure in terms of the defect arrangement and concentration, using density functional theory with the generalized gradient approximation. Our calculations demonstrate that the total energy monotonically decreases with decreasing the defect concentration, irrespective of the defect arrangements. However, the electronic structures strongly depend not only on the defect concentration but also on the defect arrangement. We observe that the bandwidth of the non-bonding $\pi$ state at the Fermi level is approximately proportional to the reciprocal of the defect spacing, which indicates the substantial penetration length of the non-bonding state associated with the defect.

\section{Calculation method}

All calculations were performed within the framework of density functional theory $[41,42]$, which was implemented in the simulation tool of atom technology (STATE) package [43]. To calculate the exchange-correlation energy among the interacting electrons, we used the generalized gradient approximation with the Perdew-Burke-Ernzerhof functional [44]. To investigate the spin-polarized states of N-doped graphene, the spin degree of freedom was taken into account in all the calculations. Vanderbilt ultrasoft pseudopotentials were used to describe the electron-ion interactions [45]. The valence wave functions and deficit charge den-

sity were expanded in terms of plane waves with cutoff energies of 25 and $225 \mathrm{Ry}$, respectively, which sufficiently describe the geometric and electronic structures of graphene and materials containing $\mathrm{N}$ atoms [46]. 

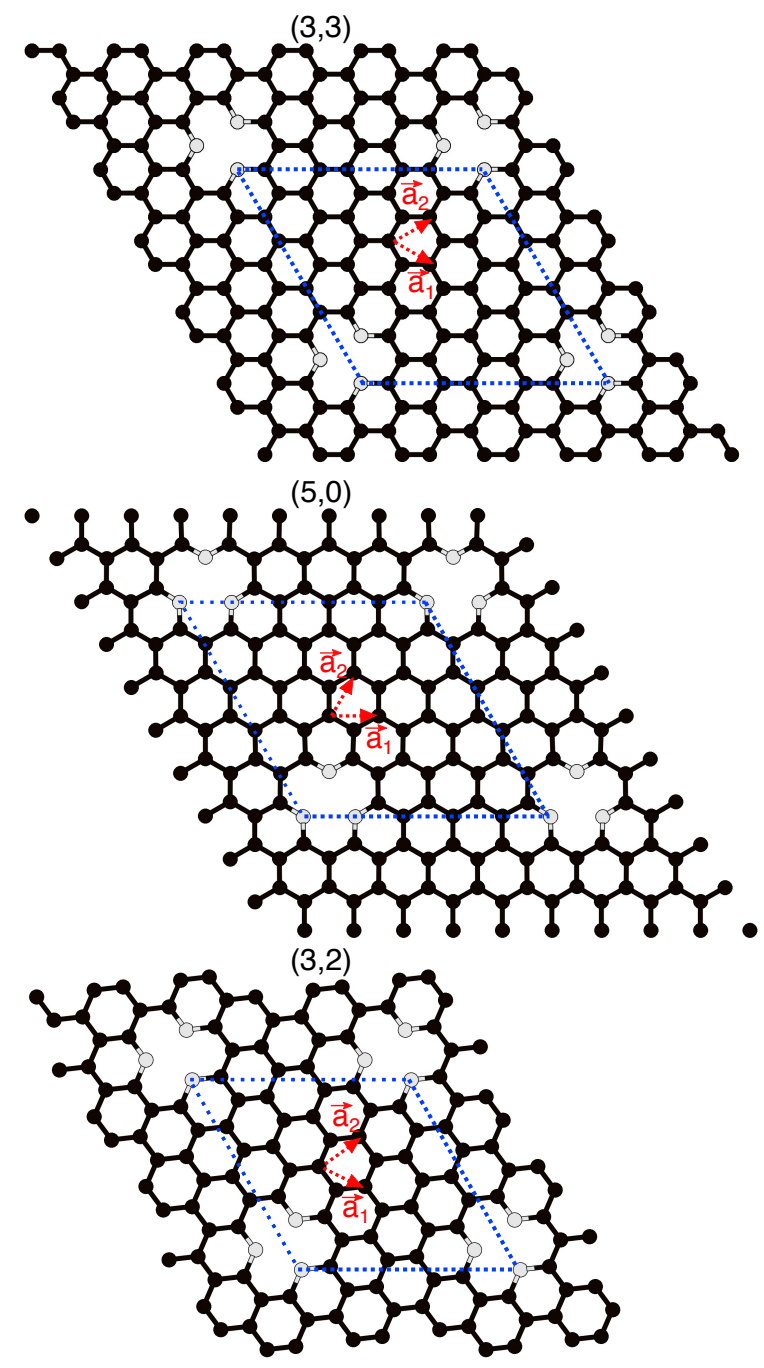

Figure 1: Optimized structures of $\mathrm{N}$-doped graphene with $(3,3),(5,0)$, and $(3,2)$ defect arrangements. Black and gray balls denote $\mathrm{C}$ and $\mathrm{N}$ atoms, respectively. Blue dotted lines and red vectors denote the unit cell and primitive vectors of $1 \mathrm{x} 1$ graphene, respectively. 
In this work, we considered graphene with monatomic defects that are surrounded by three pyridinic $\mathrm{N}$ atoms (Fig. 1). The defect density ranged from 0.006 to $0.024 / \AA^{2}$, which corresponded to the cell parameters from 6.51 to 12.78

$75 \AA$ A respectively. To investigate whether the asymptotic properties of the energetics and electronic structure depend on the mutual arrangement of the defects, we considered nine representative defect arrangements. These were characterized by a pair of integer numbers $(n, m)$, which are the coefficients of the primitive vectors, $\boldsymbol{a}_{1}$ and $\boldsymbol{a}_{2}$, of the hexagonal lattice. To simulate an isolated graphene with pyridinic defects, the defective graphene sheet was separated from its periodic images by a vacuum spacing of $10.58 \AA$. Atomic structures of the sheets were fully optimized until the force acting on each atom was less than $1.33 \times 10^{-3} \mathrm{HR} / \mathrm{au}$. Integration over the Brillouin zone was carried out using an equidistance mesh of $4 \times 4 \times 1 \boldsymbol{k}$ points, which corresponded to $12 \times 12 \times 1 \boldsymbol{k}$ points or denser for a 1 ${ }_{85} \times 1$ primitive cell of graphene.

\section{Results and discussions}

Figure 1 shows the optimized structures of three representative defective graphene sheets with indexes of $(3,3),(5,0)$, and $(3,2)$. Among the three structures, an hexagonal covalent network of the $(3,3)$ structure satisfied the Clar structure or had a full-benzenoid structure, while the other two did not satisfy the Clar structure. As for the $(n, m)$, the defect arrangements possessing the Clar structure satisfied the relation $|n-m|=3 l$, where $l$ is an integer, otherwise the networks did not satisfy this relation. Table I summarizes the lattice parameters, the bond lengths, and angles associated with the defects. The geometric structure of graphene with monovacancies surrounded by three pyridinic $\mathrm{N}$ was insensitive to the defect con- 
centration and arrangement. For all structures except $(2,1)$ and $(3,0)$, the N-C bond lengths surrounding the monovacancy were 1.33-1.34 $\AA$ and the bond angle was approximately $123^{\circ}$, irrespective of the defect density and arrangements. Additionally, the defects approximately retained a $C_{3}$ symmetry after the structural optimization. The $\mathrm{C}-\mathrm{C}$ bonds surrounding the defects were slightly elongated from those in the bulk region, because of the shorter N-C bonds. It should be noted that the bond length and angles in the $(2,1)$ structure were slightly different from the other structures, because of the small spacing between adjacent defects prohibited a sufficient structural relaxation.

Table 1: Lattice prameters $[\AA]$, optimized bond lengths $[\AA]$, and bond angles $\left[^{\circ}\right]$ of N-doped graphene with a pyridinic structure.

\begin{tabular}{cccccc}
\hline Structure & $a$ & $d_{N C}$ & $d_{C C}^{D}$ & $d_{C C}^{B}$ & $\theta_{N}$ \\
\hline$(2,1)$ & 6.51 & $1.33-1.34$ & $1.44-1.46$ & 1.43 & $123.0,123.2,123.2$ \\
$(3,0)$ & 7.38 & 1.34 & 1.44 & $1.41-1.45$ & $124.0,124.1,124.1$ \\
$(2,2)$ & 8.52 & $1.33-1.34$ & 1.45 & $1.41-1.43$ & $122.3,122.4,122.4$ \\
$(3,1)$ & 8.87 & $1.33-1.34$ & 1.45 & $1.42-1.43$ & $123.2,123.3,123.3$ \\
$(4,0)$ & 9.83 & 1.34 & 1.45 & $1.42-1.44$ & 123.0 \\
$(3,2)$ & 10.72 & 1.34 & 1.45 & $1.41-1.44$ & $122.5,122.6,122.6$ \\
$(4,1)$ & 11.27 & $1.33-1.34$ & 1.45 & $1.42-1.43$ & 123.0 \\
$(5,0)$ & 12.30 & 1.34 & 1.45 & $1.41-1.43$ & $122.8,122.9,122.9$ \\
$(3,3)$ & 12.78 & $1.33-1.34$ & 1.45 & $1.42-1.43$ & 122.7 \\
\hline
\end{tabular}

Figure 2 shows the calculated formation energy of the defects as a function of their density. The formation energy $\epsilon$ was evaluated by the following equation:

$$
\epsilon=\frac{E_{\text {total }}-3 \mu_{\mathrm{N}}-N_{\mathrm{C}} \mu_{\mathrm{C}}}{N_{\mathrm{C}}+3}
$$


where $E_{\text {total }}, N_{c}, \mu_{N}$, and $\mu_{C}$ are the total energy of $\mathrm{N}$-doped graphene, the number of $\mathrm{C}$ atoms, the chemical potential of $\mathrm{C}$, and the chemical potential of $\mathrm{N}$, respectively. The chemical potentials of $\mathrm{N}$ and $\mathrm{C}$ were evaluated by the total energies of the $\mathrm{N}_{2}$ molecule and graphene, respectively. The formation energy depended on the defect density, while it did not depend on the mutual arrangement of defects. The energy seemed to be proportional to the defect density, which indicated that the energetics were determined by the extent of the $\pi$ electrons in the graphitic area. An intercept corresponding to the dilute limit of defects indicated the energy cost to remove a $\mathrm{C}$ atom and to replace three $\mathrm{C}-\mathrm{C}$ bonds with $\mathrm{C}-\mathrm{N}$ bonds.

Figure 3 shows the electronic band structures of graphene with monovacancies surrounded by three pyridinic $\mathrm{N}$ atoms. All structures were metals where two dispersive bands and three less dispersive bands emerged near the Fermi level. Two of the three less dispersive states exhibited almost a flat band nature throughout the Brillouin zone, while the remaining state had small dispersion with a bandwidth of approximately $0.5 \mathrm{eV}$. By analyzing the wave function of these states, the small dispersion band exhibited a non-bonding $\pi$ state nature, which was distributed on one of the two sublattices containing $\mathrm{N}$ atoms. The state was localized on the defect edge at the $\mathrm{K}$ point, while it was extended throughout the sheet at the $\Gamma$ point for the Clar structure. In contrast, for the non-Clar structures, the state was extended and localized at the $\mathrm{K}$ and $\Gamma$ points, respectively. Additionally, two flat band states were localized at $\mathrm{N}$ atoms, which corresponded to the fully saturated $\sigma$ states of the pyridinic $\mathrm{N}$ atoms. Therefore, as far as the $\pi$ electron states were concerned, the electronic energy band of graphene with three pyridinic defects could be regarded as that of graphene with a monovacancy, where the $p_{z}$ orbital of the pyridinic $\mathrm{N}$ participates in the $\pi$ states of graphene, because of their isovalence 


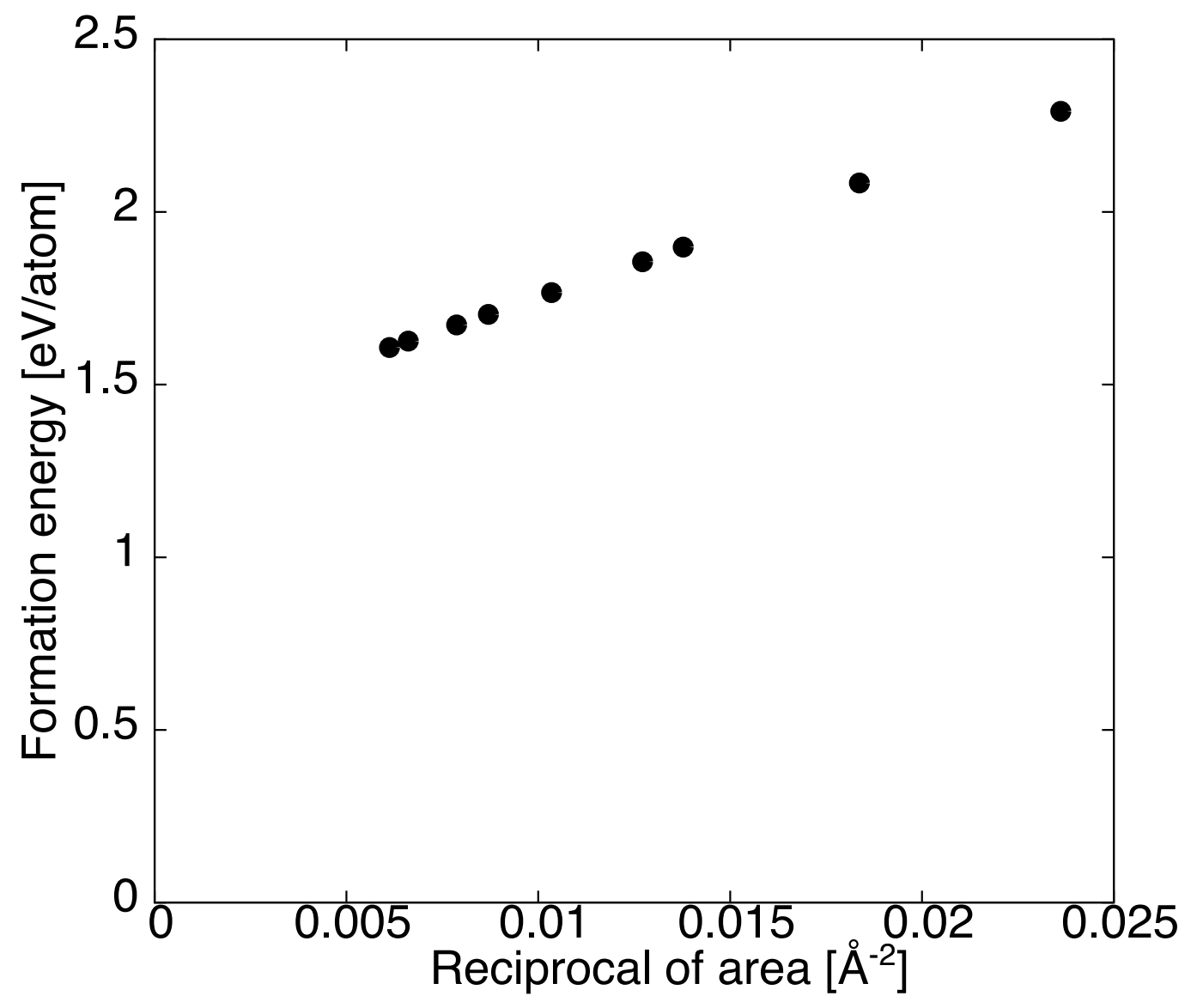

Figure 2: Formation energy of defects as a function of the defect density $\left(/ \AA^{2}\right)$. 
to an $\mathrm{sp}^{2} \mathrm{C}$ atom.

By focusing on the $\pi$ electron states of graphene with pyridinic defects, the electronic structure can be classified into two groups. As for the $(3,3)$ structure whose hexagonal network satisfies Clar structure, two substantial dispersive bands had a small gap of $0.05 \mathrm{meV}$ at the $\Gamma$ point, which formed a massive Dirac cone [Fig. 3(a)]. Furthermore, the less dispersive $\pi$ state associated with the nonbonding state was degenerate with the bonding $\pi$ state at the $\Gamma$ point. The characteristic electronic band structure around the $\Gamma$ point could be interpreted as one of the two Dirac cones at the K point of the primitive cell of graphene is folded into the $\Gamma$ point and the sublattice imbalance induced by the monovacancy causes the non-bonding states with a narrow bandwidth. Furthermore, although the $p_{z}$ state of pyridinic $\mathrm{N}$ naively acts as the $\pi$ state of an $\mathrm{sp}^{2} \mathrm{C}$ atom, the deeper atomic potential of $\mathrm{N}$ than that of $\mathrm{C}$ causes a smaller onsite energy for the majority of the sublattice including $\mathrm{N}$ atoms and opens the tiny gap between bonding and antibonding $\pi$ states at the $\Gamma$ point. Accordingly, the wave function of the highest branch of the valence band and the lowest branch of the conduction band at the $\Gamma$ point exhibited a non-bonding nature, as is the case of pristine graphene at the $\mathrm{K}$ point. In contrast, at the $\mathrm{K}$ point, the less dispersive band exhibited a non-bonding and localized nature around the defects.

For defective graphene with $(5,0)$ and $(3,2)$ pyridinic $\mathrm{N}$ arrangements, dispersive $\pi$ bands had a large gap of approximately $1.5 \mathrm{eV}$ at the $\mathrm{K}$ point. In this case, the hexagonal networks consisting of $\mathrm{C}$ and pyridinic $\mathrm{N}$ did not satisfy the Clar structure, which resulted in the mixing of the $\pi$ wave functions belonging to different sublattices. According to the wave function mixing, the wave functions at the $\mathrm{K}$ point could not render a non-bonding $\pi$ state as that in pristine graphene, 
and graphene with pyridinic $\mathrm{N}$ had a large gap at the $\mathrm{K}$ point. Indeed, the top of the valence and the bottom of the conduction bands at the $\mathrm{K}$ point had their wave function distribution on both sublattices. As for the less dispersive band associated with the non-bonding state, which arose from the sublattice imbalance, the state was localized around the defect at the $\Gamma$ point, while it extended throughout the network at the $\mathrm{K}$ point.

The energy gap between bonding and antibonding $\pi$ states, as well as the whole electronic structure of graphene with monovacancies surrounded by pyridinic $\mathrm{N}$, depend on whether or not the hexagonal networks of $\mathrm{C}$ and $\mathrm{N}$ satisfy the Clar structure. Figure 4 shows the $\pi-\pi^{*}$ gap of graphene with pyridinic N defects as a function of the reciprocal of the defect spacing. The gap depended not only on the mutual defect arrangement but also on the defect spacing. The gap decreased with increasing the defect spacing for the defects with and without the Clar structures. For graphene with pyridinic $\mathrm{N}$ defects that satisfied the Clar structure, the gap was approximately proportional to the reciprocal of the defect spacing: The gaps were 0.14 and $0.05 \mathrm{eV}$ for the defect arrangements $(3,0)$ and $(3,3)$, respectively. For the non-Clar structures, the gap exhibited a further dependence on the defect arrangements. The defect arrangement $(n, m)$ satisfied the relation $|n-m|=3 l+1$, where $l$ is an integer and has slightly narrower $\pi-\pi^{*}$ gap than that satisfying $|n-m|=3 l+2$. For these defect arrangements, the gap ranged from $3 \mathrm{eV}$ for the defect spacing of $6.51 \AA$ to $1.5 \mathrm{eV}$ for the defect spacing of $12.30 \AA$.

Finally, we determined how the bandwidth of the non-bonding state at the 180 Fermi level depends on the defect arrangement and density. The charge localization, owing to the flat dispersion band, naively increased the reactivity at the 
(a)
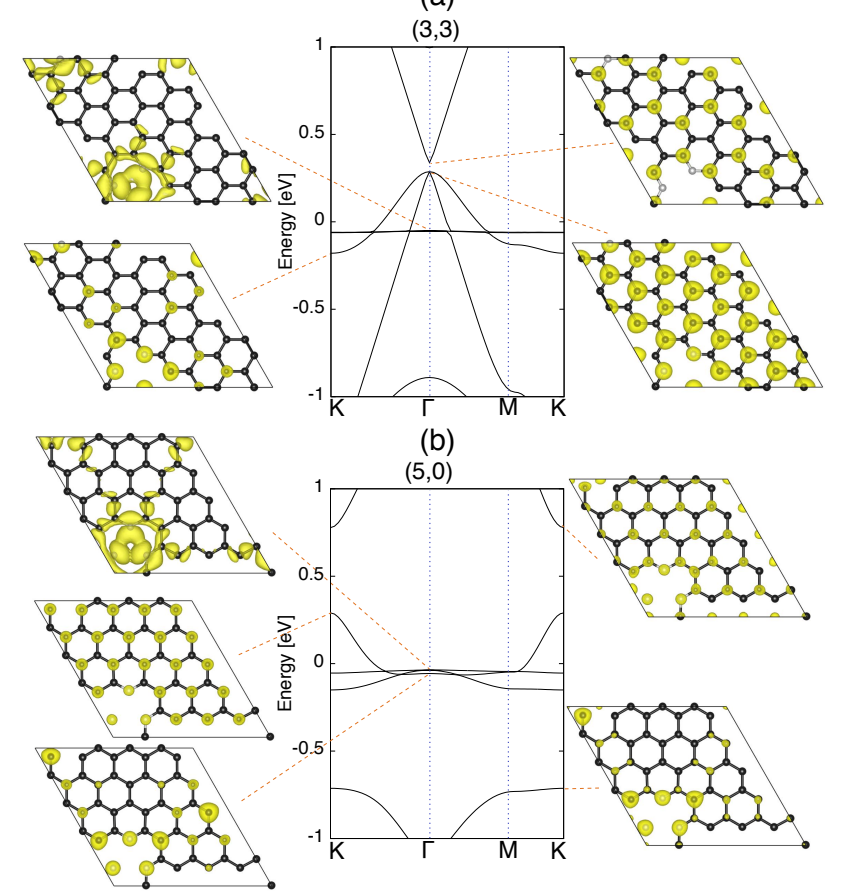

(b)

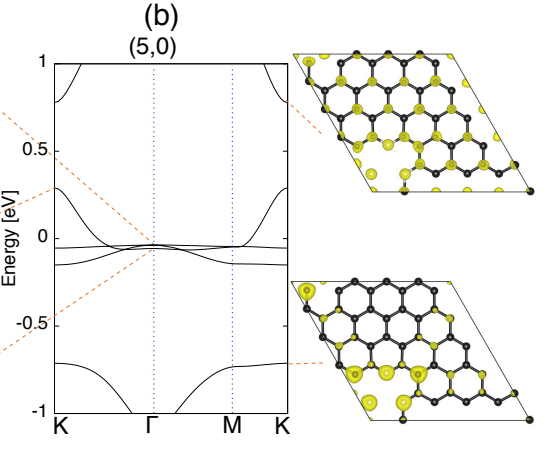

(c)

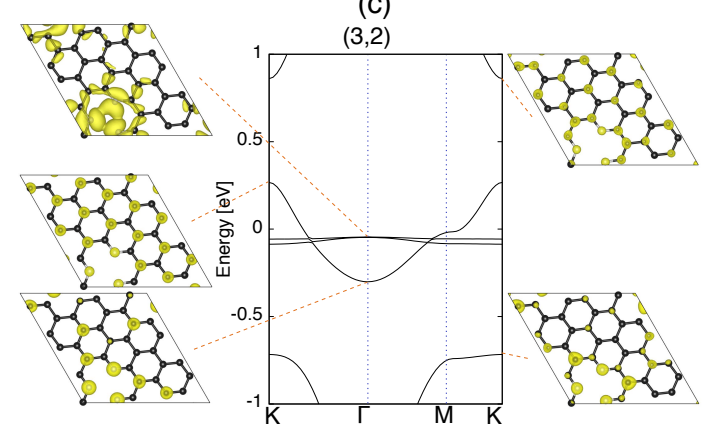

Figure 3: Electronic band structures and squared wave functions near the Fermi level at the $\mathrm{K}$ and $\Gamma$ points of $\mathrm{N}$-doped graphene with (a) $(3,3)$, (b) $(5,0)$, and (c) $(3,2)$ defect arrangements. 


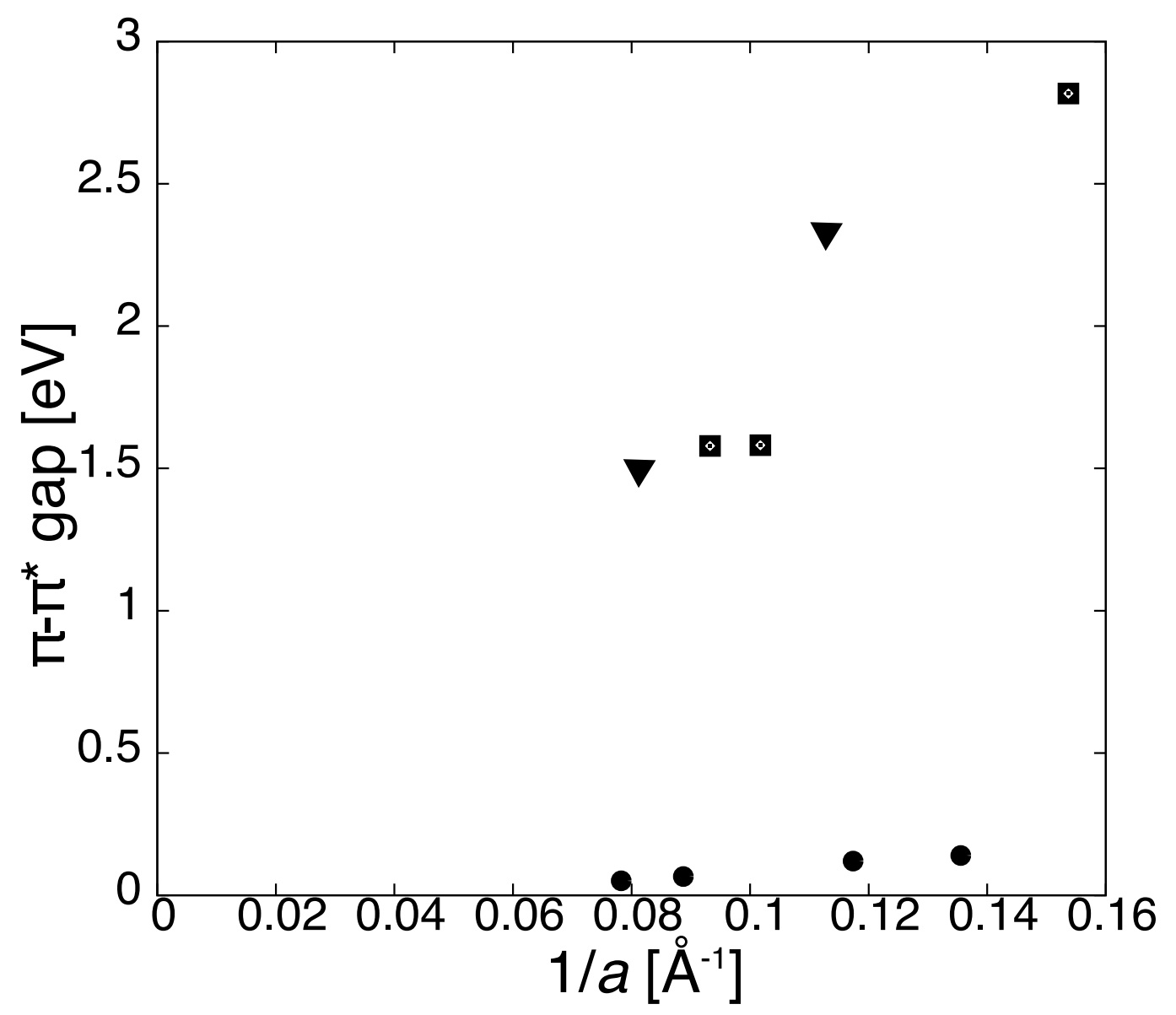

Figure 4: Energy gap between bonding and antibonding $\pi$ states of graphene with monovacancy surrounded by pyridinic $\mathrm{N}$ as a function of the reciprocal of the defect spacing. Circles, squares, and triangles correspond to the structures of $|n-m|=3 l,|n-m|=3 l+1$, and $|n-m|=3 l+2$, respectively. 
particular sites where electrons localized, which caused the catalytic activity of graphene with pyridinic defects. Figure 5 shows the bandwidth of the non-bonding state near the Fermi level as a function of the reciprocal of the defect-defect spacing. The gap basically decreased with increasing the defect-defect spacing for all defect arrangements. In addition, the width also depended on the defect arrangements. The defect arrangements of $|n-m|=3 l+2$ had a relatively large width, while the arrangement of $|n-m|=3 l+1$ had a small width. The defect arrangement with $|n-m|=3 l$ was marginal. The bandwidth seemed to asymptotically approach zero with the increase of the defect-defect spacing, and it was expected to still be $0.1 \mathrm{eV}$ for the case where the defect was separated from its adjacents by $5 \mathrm{~nm}$. The substantial band dispersion of the non-bonding state at the Fermi levels arose from the delocalized nature of the wave function associated with the states at the $\Gamma$ and $\mathrm{K}$ points for the defect arrangements with and without Clar structure, respectively, as shown in Fig. 3. The narrow but significant bandwidth of the non-bonding states caused the usual phenomena associated with the crossover between strong and weak electron correlations as was observed for the case of the electron-doped solid $\mathrm{C}_{60}$, which has a bandwidth of a few hundred meV at the Fermi level. In particular, the coupling between the $\pi$ electron on networks with weakly itinerated nature and the dangling bond states of $\mathrm{N}$ with strong localized nature caused the peculiar electron transport, where the itinerant electron was scattered by a localized electron at the defect sites.

\section{Conclusion}

Using density functional theory with the generalized gradient approximation, we investigated the energetics and electronic structure of graphene with monova- 


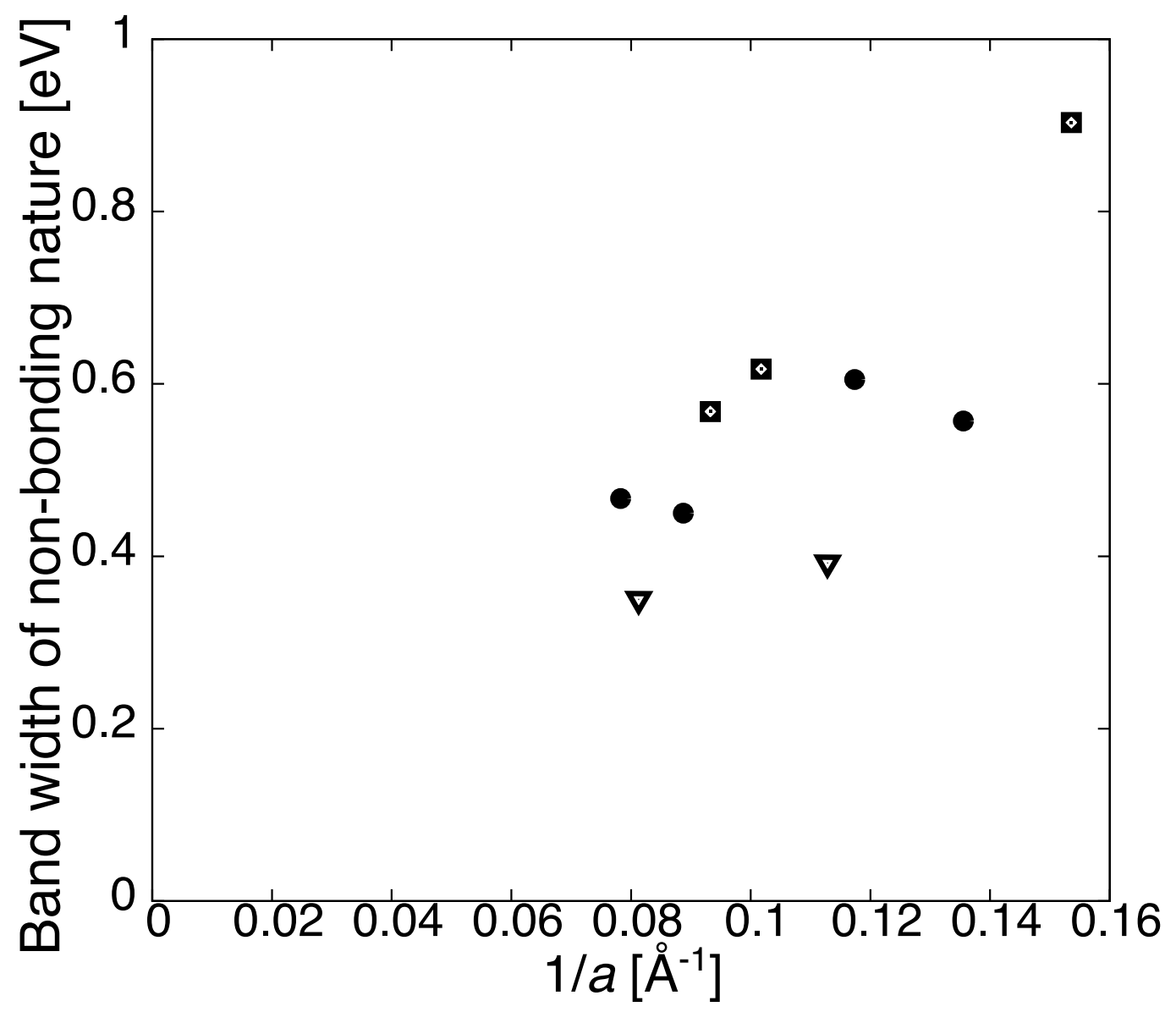

Figure 5: Bandwidth of the non-bonding state at the Fermi level as a function of the reciprocal of the defect spacing. Circles, squares, and triangles correspond to the structures of $|n-m|=3 l$, $|n-m|=3 l+1$, and $|n-m|=3 l+2$, respectively. 
cancy, whose edges are terminated by $\mathrm{N}$ atoms with pyridinic structure, in terms of the defect arrangement and concentration. Our calculations demonstrated that the total energy monotonically decreases with decreasing the defect density, irrespective of the defect arrangements. However, the electronic structures strongly depend not only on the defect density but also on the defect arrangement. We observed that the bandwidth of the non-bonding $\pi$ state was approximately proportional to the defect-defect spacing, indicating the substantial penetration length of the non-bonding $\pi$ state associated with the defect.

\section{Acknowledgement}

The authors acknowledge JST-CREST Grant Numbers JPMJCR1532 and JPMJCR1715 from the Japan Science and Technology Agency, JSPS KAKENHI Grant Numbers JP17H01069, JP16H00898, and JP16H06331 from the Japan Society for the Promotion of Science, and the Joint Research Program on ZeroEmission Energy Research, Institute of Advanced Energy, Kyoto University. Part of the calculations was performed on an NEC SX-Ace at the Cybermedia Center at Osaka University and on an SGI ICE XA/UV at the Institute of Solid State Physics, The University of Tokyo.

\section{References}

[1] F. Bassani and G.P. Parravioini, Band structure and optical properties of graphite and of the later compounds GaS and GaSe, Il Nuovo Cimento B Series 1050 (1967) 95-128.

[2] G.S. Painter and D.E. Ellis, Electronic band structure and optical properties of graphite from a variational approach, Phys. Rev. B 1 (1970) 4747-4752. 
[3] A.J. Freeman, E. Wimmer, and M. Weinert, Prediction of electronic interlayer states in graphite and reinterpretation of alkali bands in graphite intercalation compounds, Phys. Rev. Lett. 50 (1983) 761-764.

[4] K.S. Kim, Y. Zhao, H. Jang, S.Y. Lee, J.M. Kim, K.S. Kim, et al., Largescale pattern growth of graphene films for stretchable transparent electrodes, Nature 457 (2009) 706-710.

[5] Y. Zhang, Y.-W. Tan, H.L. Stormer, and P. Kim, Experimental observation of the quantum Hall effect and Berry's phase in graphene, Nature 438 (2005) 201-204.

[6] M.F. Craciun, S. Russo, M. Yamamoto, J.B. Oostinga, A.F. Morpurgo, and S. Tarucha, Trilayer graphene is a semimetal with a gate-tunable band overlap, Nature Nanotech. 4 (2009) 383-388.

[7] T. Ohta, A. Bostwick, T. Seyller, K. Horn, and E. Rotenberg, Controlling the electronic structure of bilayer graphene, Science 313 (2006) 951-954.

[8] S.Y. Zhou, G.-H. Gweon, A.V. Fedorov, P.N. First, W.A. de Heer, D.-H. Lee, et al., Substrate-induced bandgap opening in epitaxial graphene, Nature Mater. 6 770-775 (2007).

[9] A. Mattausch and O. Pankratov, Ab initio study of graphene on SiC, Phys. Rev. Lett. 99 (2007) 076802.

[10] N.-T. Cuong, M. Otani, and S. Okada, Semiconducting electronic property of graphene adsorbed on (0001) Surfaces of $\mathrm{SiO}_{2}$, Phys. Rev. Lett. 106 (2011) 106801 . 
[11] K. Kamiya, N. Umezawa, and S. Okada, Energetics and electronic structure of graphene adsorbed on $\mathrm{HfO}_{2}(111)$ : Density functional theory calculations, Phys. Rev. B 83 (2011) 153413.

[12] M. Fujita, K. Wakabayashi, K. Nakada, and K. Kusakabe, Peculiar localized state at zigzag graphite edge, J. Phys. Soc. Jpn. 65 (1996) 1920-1923.

[13] K. Nakada, M. Fujita, G. Dresselhaus, and M.S. Dresselhaus, Edge state in graphene ribbons: Nanometer size effect and edge shape dependence, Phys. Rev. B 54 (1996) 17954-17961.

[14] Y.-W. Son, M.L. Cohen, and S.G. Louie, Energy gaps in graphene nanoribbons, Phys. Rev. Lett. 97 (2006) 216803.

[15] Y. Miyamoto, K. Nakada, and M. Fujita, First-principles study of edge states of H-terminated graphitic ribbons, Phys. Rev. B 59 (1999) 9858-9861.

[16] Y. Kobayashi, K. Fukui, and T. Enoki, Edge state on hydrogen-terminated graphite edges investigated by scanning tunneling microscopy, Phys. Rev. B 73 (2006) 125415.

[17] A. Yamanaka and S. Okada, Energetics and electronic structures of graphene nanoribbons under a lateral electric field, Carbon 96 (2016) 351-361.

[18] S. Bhowmick, Weber-fechner type nonlinear behavior in zigzag edge graphene nanoribbons, Phys. Rev. B 82 (2010) 155448.

[19] S. Bhowmick, A. Medhi, and V.B. Shenoy, Sensory-organ-like response determines the magnetism of zigzag-edged honeycomb nanoribbons, Phys. Rev. B 87 (2013) 085412. 
[20] K. Kusakabe, K. Wakabayashi, M. Igami, K. Nakada, and M. Fujita, Magnetism of nanometer-scale graphite with edge or topological defects, Mol. Cryst. Liq. Cryst. 305 (1997) 445-454.

[21] M. Maruyama and S. Okada, A two-dimensional $\mathrm{sp}^{2}$ carbon network of fused pentagons: All carbon ferromagnetic sheet, Appl. Phys. Express 6 (2013) 095101.

[22] S. Okada, T. Kawai, and K. Nakada, Electronic structure of graphene with topological line defect, J. Phys. Soc. Jpn. 80 (2011) 013709.

[23] J. Fernández-Rossier and J.J. Palacios, Magnetism in graphene nanoislands, Phys. Rev. Lett. 99 (2007) 177204.

[24] N. Shima and H. Aoki, Electronic structure of super-honeycomb systems: A peculiar realization of semimetal/semiconductor classes and ferromagnetism, Phys. Rev. Lett. 71 (1993) 4389-4392.

[25] M. Maruyama and S. Okada, Coexistence of Dirac cones and Kagome flat bands in a porous graphene, Carbon 109 (2016) 755-763.

[26] M. Igami, S. Okada, ad K. Nakada, Electronic and geometric structures of fluorine adsorbed graphene Synth. Met. 1 (2001) 1233-1234.

[27] T. Matsumoto and S. Saito, J. Phys. Soc. Jpn. 71 (2002) 2765-2770.

[28] H. Jippo, M. Ohfuchi, and C. Kaneta, Phys. Rev. B 84 (2011) 075467.

[29] H. Sawahata, M. Maruyama, N.-T. Cuong, H. Omachi, H. Shinohara, and S. Okada, Band-gap engineering graphene heterostructures by substitutional doping with $\mathrm{B}_{3} \mathrm{~N}_{3}$, ChemPhysChem 19 (2018) 237-242. 
[36] Z. Hou, Z. Wang, T. Ikeda, K. Terakura, M. Oshima, M. Kakimoto, Interplay between nitrogen dopants and native point defects in graphene, Phys. Rev. B 85 (2012) 165439.

[37] T. Susi, J. Kotakoski, R. Arenal, S. Kurasch, H. Jiang, V. Skakalova, Atomistic description of electron beam damage in nitrogen-doped graphene and single-walled carbon nanotube, ACS Nano 6 (2012) 8837-8846. 
[38] Y. Fujimoto and S. Saito, Hydrogen adsorption and anomalous electronic properties of nitrogen-doped graphene, Appl. Phys. Lett. 115 (2014) 153701.

[39] T. Kondo, T. Suzuki, and J. Nakamura, Nitrogen doping of graphite for enhancement of durability of supported platinum clusters, J. Phys. Chem. Lett. 2 (2011) 577-580.

[40] T. Kondo, S. Casolo, T. Suzuki, T. Shikano, M. Sakurai, Y. Harada, et al., Atomic-scale characterization of nitrogen-doped graphite: Effects of dopant nitrogen on the local electronic structure of the surrounding carbon atoms, Phys. Rev. B 86 (2012) 035436.

[41] P. Hohenberg, W. Kohn, Inhomogeneous electron gas, Phys. Rev. 136 (1964) B864-B871.

[42] W. Kohn, L.J. Sham, Self-consistent equations including exchange and correlation effects, Phys. Rev. 40 (1965) A1133-A1138.

${ }_{330}$ [43] Y. Morikawa, K. Iwata, K. Terakura, Theoretical study of hydrogenation process of formate on clean and $\mathrm{Zn}$ deposited $\mathrm{Cu}(111)$ surfaces, Appl. Surf. Sci. 169-170 (2001) 11-15.

[44] J.P. Perdew, K. Burke, M. Ernzerhof, Generalized gradient approximation made simple, Phys. Rev. Lett. 77 (1996) 3865-3868.

[45] D. Vanderbilt, Soft self-consistent pseudopotentials in a generalized eigenvalue formalism, Phys. Rev. B 41 (1990) 7892-7895.

[46] M. Maruyama and S. Okada, Magnetic properties of graphene quantum dots embedded in h-BN sheet, J. Phys. Chem. C 120 (2016) 1293-1302. 